Supplement of Geosci. Model Dev., 9, 1201-1218, 2016

http://www.geosci-model-dev.net/9/1201/2016/

doi:10.5194/gmd-9-1201-2016-supplement

(C) Author(s) 2016. CC Attribution 3.0 License.

(c) (1)

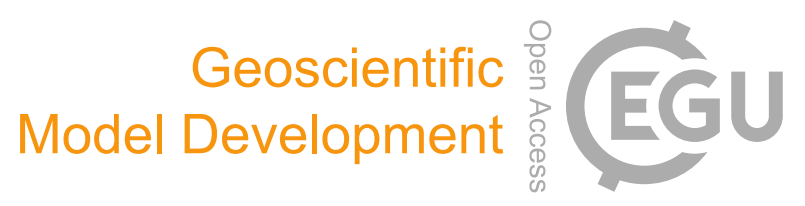

Supplement of

\title{
Air quality modeling with WRF-Chem v3.5 in East Asia: sensitivity to emissions and evaluation of simulated air quality
}

Min Zhong et al.

Correspondence to: Min Zhong (min.zhong@emory.edu) and Eri Saikawa (eri.saikawa@emory.edu)

The copyright of individual parts of the supplement might differ from the CC-BY 3.0 licence. 
Table S1. Ratios used to create hourly emissions for each pollutant in this study. The diurnal variation is adapted from the East Asian Air pollutant Emission Grid Database (EAGrid2000,

http://www.cger.nies.go.jp/db/eagrid/eagrid_index_e.html)

\begin{tabular}{ccccccc}
\hline Hours & $\mathbf{C O}$ & NO$_{\mathbf{x}}$ & $\mathbf{N M V O C}$ & $\mathbf{S O}_{\mathbf{2}}$ & $\mathbf{N H}_{\mathbf{3}}$ & $\mathbf{P M} / \mathbf{B C} \mathbf{O C}$ \\
\hline 0 & 0.015 & 0.023 & 0.019 & 0.033 & 0.021 & 0.021 \\
1 & 0.015 & 0.023 & 0.019 & 0.033 & 0.021 & 0.021 \\
2 & 0.015 & 0.023 & 0.019 & 0.033 & 0.021 & 0.021 \\
3 & 0.015 & 0.023 & 0.019 & 0.033 & 0.021 & 0.021 \\
4 & 0.015 & 0.023 & 0.023 & 0.033 & 0.021 & 0.025 \\
5 & 0.030 & 0.031 & 0.023 & 0.033 & 0.025 & 0.031 \\
6 & 0.038 & 0.046 & 0.028 & 0.038 & 0.029 & 0.040 \\
7 & 0.075 & 0.061 & 0.056 & 0.038 & 0.038 & 0.048 \\
8 & 0.068 & 0.061 & 0.075 & 0.043 & 0.042 & 0.063 \\
9 & 0.060 & 0.061 & 0.075 & 0.047 & 0.050 & 0.063 \\
10 & 0.060 & 0.061 & 0.071 & 0.052 & 0.067 & 0.062 \\
11 & 0.057 & 0.054 & 0.066 & 0.052 & 0.084 & 0.060 \\
12 & 0.057 & 0.058 & 0.066 & 0.052 & 0.092 & 0.058 \\
13 & 0.060 & 0.058 & 0.066 & 0.052 & 0.084 & 0.062 \\
14 & 0.060 & 0.058 & 0.066 & 0.052 & 0.076 & 0.060 \\
15 & 0.060 & 0.058 & 0.056 & 0.052 & 0.063 & 0.060 \\
16 & 0.064 & 0.054 & 0.056 & 0.047 & 0.050 & 0.058 \\
17 & 0.060 & 0.046 & 0.053 & 0.043 & 0.042 & 0.046 \\
18 & 0.042 & 0.038 & 0.028 & 0.043 & 0.034 & 0.042 \\
19 & 0.034 & 0.031 & 0.028 & 0.043 & 0.025 & 0.035 \\
20 & 0.030 & 0.031 & 0.023 & 0.040 & 0.025 & 0.029 \\
21 & 0.026 & 0.028 & 0.023 & 0.038 & 0.025 & 0.027 \\
22 & 0.026 & 0.025 & 0.023 & 0.036 & 0.021 & 0.025 \\
23 & 0.015 & 0.023 & 0.023 & 0.033 & 0.021 & 0.023 \\
\hline & & & & & &
\end{tabular}


Table S2: Air Pollution Index (API) and corresponding daily mean $\mathrm{PM}_{10}$ concentrations (Source: http://jcs.mep.gov.cn/hjzl/200604/t20060426 76155.htm).

\begin{tabular}{cc}
\hline API & Daily mean $\mathrm{PM}_{10}\left(\mathrm{ug} \mathrm{m}^{-3}\right)$ \\
\hline 50 & 50 \\
100 & 150 \\
200 & 350 \\
300 & 420 \\
400 & 500 \\
500 & 600 \\
\hline
\end{tabular}


Table S3a. Description of API derived PM $_{10}$ observation sites from the website of Ministry of Environmental Protection of P. R. China, http://datacenter.mep.gov.cn/

\begin{tabular}{|c|c|c|c|c|}
\hline Site & Province & Region & Latitude $\left({ }^{\circ} \mathrm{N}\right)$ & Longitude $\left({ }^{\circ} \mathbf{E}\right)$ \\
\hline Anshan & Liaoning & Northeast & 41.11 & 122.99 \\
\hline Baoji & Shanxi & North & 34.36 & 107.24 \\
\hline Beihai & Guangxi & South & 21.48 & 109.12 \\
\hline Beijing & Beijing & North & 39.9 & 116.4 \\
\hline Changchun & Jilin & Northeast & 43.83 & 125.32 \\
\hline Changde & Hunan & Central & 29.03 & 111.7 \\
\hline Changsha & Hunan & Central & 28.23 & 112.94 \\
\hline Changzhi & Shanxi & North & 36.2 & 113.12 \\
\hline Chengdu & Sichuang & Southwest & 30.57 & 104.06 \\
\hline Chifeng & Neimengg & North & 42.26 & 118.89 \\
\hline Chongqin & Chongqin & Southwest & 29.56 & 106.55 \\
\hline Dalian & Liaoning & Northeast & 38.91 & 121.62 \\
\hline Datong & Shanxi & North & 40.07 & 113.3 \\
\hline Deyang & Sichuang & Southwest & 31.12 & 104.4 \\
\hline Fushun & Liaoning & Northeast & 41.88 & 123.96 \\
\hline Guangzhou & Guangdon & South & 23.13 & 113.26 \\
\hline Guiyan & Guizhou & Southwest & 26.65 & 106.63 \\
\hline Haerbin & Heilongj & Northeast & 45.8 & 126.54 \\
\hline Haikou & Hainan & South & 20.02 & 110.33 \\
\hline Hangzhou & Zhejiang & East & 30.27 & 120.16 \\
\hline Hefei & Anhui & East & 31.82 & 117.23 \\
\hline Huhehot & Neimengg & North & 40.84 & 111.75 \\
\hline Jinan & Shandong & East & 36.65 & 117.12 \\
\hline Jingzhou & Hubei & Central & 30.33 & 112.24 \\
\hline Jining & Shandong & East & 35.42 & 116.59 \\
\hline Jiujiang & Jiangxi & East & 29.71 & 116 \\
\hline Kaifeng & Henan & Central & 34.8 & 114.31 \\
\hline Kunmin & Yunnan & Southwest & 24.88 & 102.83 \\
\hline Lanzhou & Gansu & Northwest & 36.06 & 103.84 \\
\hline Lhasa & Xizang & Southwest & 29.65 & 91.14 \\
\hline Liuzhou & Guangxi & South & 24.32 & 109.42 \\
\hline Luzhou & Sichuang & Southwest & 28.87 & 105.44 \\
\hline Mianyan & Sichuang & Southwest & 31.46 & 104.68 \\
\hline Mudanjiang & Heilongj & Northeast & 44.55 & 129.63 \\
\hline Nanchang & Jiangxi & East & 28.68 & 115.86 \\
\hline Nanchong & Sichuang & Southwest & 30.83 & 106.11 \\
\hline Nanjing & Jiangsu & East & 32.06 & 118.8 \\
\hline Nanning & Guangxi & South & 22.82 & 108.37 \\
\hline Ningbo & Zhejiang & East & 29.86 & 121.54 \\
\hline Pingding & Henan & Central & 33.77 & 113.19 \\
\hline Qingdao & Shandong & East & 36.08 & 120.38 \\
\hline Qiqihaer & Heilongj & Northeast & 47.35 & 123.92 \\
\hline
\end{tabular}




\begin{tabular}{|c|c|c|c|c|}
\hline Quanzhou & Fujian & East & 24.87 & 118.68 \\
\hline Guilin & Guangxi & South & 25.27 & 110.29 \\
\hline Qujing & Yunnan & Southwest & 25.49 & 103.8 \\
\hline Rizhao & Shandong & East & 35.47 & 119.4 \\
\hline Shanghai & Shanghai & East & 31.22 & 121.46 \\
\hline Shantou & Guangdon & South & 23.29 & 116.54 \\
\hline Shaoguan & Guangdon & South & 24.81 & 113.6 \\
\hline Shenyang & Liaoning & Northeast & 41.81 & 123.43 \\
\hline Shenzhen & Guangdon & South & 22.54 & 114.06 \\
\hline Shijiazhuang & Hebei & North & 38.04 & 114.5 \\
\hline Shizuishan & Ningxia & Northwest & 38.98 & 106.38 \\
\hline Taian & Shandong & East & 36.2 & 117.09 \\
\hline Tianjin & Tianjin & North & 39.2 & 117.4 \\
\hline Weifang & Shandong & East & 36.71 & 119.16 \\
\hline Weinan & Shanxi & North & 34.5 & 109.51 \\
\hline Wuhan & Hubei & Central & 30.59 & 114.31 \\
\hline Wuhu & Anhui & East & 31.35 & 118.43 \\
\hline Urumqi & Xinjiang & Northwest & 43.83 & 87.62 \\
\hline Xiamen & Fujian & East & 24.52 & 118 \\
\hline Xian & Shanxi & North & 34.25 & 109.09 \\
\hline Xining & Qinghai & Northwest & 36.62 & 101.78 \\
\hline Yangquan & Shanxi & North & 37.86 & 113.58 \\
\hline Yantai & Shandong & East & 37.46 & 121.44 \\
\hline Yinchuan & Ningxia & Northwest & 38.49 & 106.23 \\
\hline Zaozhuang & Shandong & East & 34.81 & 117.32 \\
\hline Zhanjiang & Guangdon & South & 21.26 & 110.36 \\
\hline Zhengzhou & Henan & Central & 34.74 & 113.63 \\
\hline Zibo & Shandong & East & 36.81 & 118.06 \\
\hline Zigong & Sichuang & Southwest & 29.34 & 104.78 \\
\hline
\end{tabular}


Table S3b. Description of observation sites with direct measurements.

\begin{tabular}{cccc}
\hline Site & Country or Region & Latitude $\left({ }^{\mathbf{0}} \mathbf{N}\right)$ & Longitude $\left({ }^{\mathbf{0}} \mathbf{E}\right)$ \\
\hline Lulin $^{1}$ & Taiwan & 23.46 & 120.87 \\
Happo $^{1}$ & Japan & 36.68 & 137.8 \\
Hedo & Japan & 26.85 & 128.26 \\
Oki & Japan & 36.28 & 133.18 \\
Rishiri & Japan & 45.12 & 141.24 \\
Sado-seki & Japan & 38.25 & 138.4 \\
Tappi & Japan & 41.27 & 141.35 \\
Yusuhara & & 33.38 & 132.93 \\
Xiamen & Japan & 24.467 & 118.133 \\
Jinyunshan $^{1}$ & China & 29.820 & 106.382 \\
Zhuhai $_{\text {Godavari }}$ & China & 22.267 & 113.567 \\
\hline
\end{tabular}

Note1: The altitude is $2860 \mathrm{~m}$ for Lulin, $1850 \mathrm{~m}$ for Happo, $790 \mathrm{~m}$ for Yusuhara, and $800 \mathrm{~m}$ for Jinyunshan. 
Table S4. Provincial differences of monthly emissions and provincial difference of 14-day mean concentrations between REAS and EDGAR in July 2007.

\begin{tabular}{|c|c|c|c|c|c|c|c|c|}
\hline \multirow{2}{*}{ Provinces in China } & \multicolumn{4}{|c|}{ Emission difference (REAS-EDGAR) / EDGAR (\%) } & \multicolumn{4}{|c|}{ Concentration difference (REAS-EDGAR) / EDGAR (\%) } \\
\hline & $\mathrm{PM}_{10}$ & $\mathrm{CO}$ & $\mathrm{SO}_{2}$ & $\mathrm{NO}_{\mathrm{x}}$ & $\mathrm{PM}_{10}$ & $\mathrm{O}_{3}$ & $\mathrm{SO}_{2}$ & $\mathrm{NO}_{2}$ \\
\hline Anhui & 61.8 & 56.4 & -26.2 & 50.6 & 48.0 & 18.0 & -28.0 & 43.7 \\
\hline Beijing & 98.4 & 238.1 & -42.3 & 32.0 & 43.8 & 19.5 & -21.1 & 27.1 \\
\hline Chongqing & 46.1 & 57.8 & 78.5 & 59.5 & 39.3 & 19.6 & 69.3 & 46.7 \\
\hline Fujian & -10.5 & 62.0 & -49.9 & 74.6 & 8.5 & 11.8 & -54.1 & 60.1 \\
\hline Guangdong & 43.0 & 67.0 & -20.2 & 49.0 & 14.2 & 9.5 & -25.7 & 51.0 \\
\hline Gansu & 37.1 & 24.7 & -31.7 & 27.6 & 1.7 & 13.6 & -22.2 & 28.6 \\
\hline Guangxi & 23.7 & 141.9 & 45.8 & 21.2 & 15.8 & 9.4 & 45.0 & 28.3 \\
\hline Guizhou & 21.5 & 205.7 & 244.2 & 68.9 & 25.1 & 13.7 & 220.8 & 58.7 \\
\hline Hainan & -10.2 & 19.8 & 11.4 & 60.5 & -1.1 & -8.4 & 13.6 & 61.8 \\
\hline Hebei & 150.6 & 548.6 & 45.2 & 92.8 & 48.2 & 20.7 & 28.0 & 65.9 \\
\hline Henan & 135.0 & 104.7 & 22.2 & 107.3 & 66.6 & 17.8 & 22.5 & 95.2 \\
\hline Heilongjiang & 9.9 & 90.0 & -51.4 & 65.8 & 17.0 & 13.3 & -52.3 & 57.7 \\
\hline Hunan & 73.4 & 92.9 & 5.1 & 59.0 & 25.4 & 18.7 & 1.5 & 53.3 \\
\hline Hubei & 6.0 & -49.9 & 44.9 & 76.6 & 41.9 & 23.8 & 15.1 & 51.2 \\
\hline Jilin & 51.9 & 131.4 & -13.2 & 109.0 & 43.0 & 19.2 & -1.3 & 111.9 \\
\hline Jiangsu & 74.2 & 311.8 & -3.0 & 108.1 & 45.9 & 13.0 & -5.4 & 95.6 \\
\hline Jiangxi & 84.8 & 57.0 & 11.3 & 36.6 & 25.6 & 20.0 & 6.9 & 54.5 \\
\hline Liaoning & 80.7 & 14.4 & 1.6 & 68.5 & 43.9 & 16.2 & -8.4 & 52.5 \\
\hline Inner Mongolia & -0.4 & 11.4 & -57.4 & 37.7 & 4.3 & 13.9 & -47.5 & 32.1 \\
\hline Ningxia & 41.5 & 84.4 & 62.6 & 86.5 & 4.2 & 13.2 & 25.9 & 66.3 \\
\hline Qinghai & 3.2 & -48.0 & -59.2 & -44.8 & -5.6 & 4.2 & -45.9 & -41.9 \\
\hline Shaanxi & 8.3 & 29.5 & -4.4 & 16.6 & 20.9 & 16.1 & -8.6 & 11.9 \\
\hline Sichuan & 33.9 & 94.0 & -1.9 & 11.0 & 25.0 & 11.0 & 6.9 & 13.7 \\
\hline Shandong & 118.6 & 344.8 & 98.1 & 143.9 & 70.3 & 19.9 & 75.3 & 118.1 \\
\hline Shanghai & 36.2 & 340.8 & 55.9 & 193.5 & 33.9 & 1.3 & 67.2 & 258.2 \\
\hline Shanxi & 160.1 & 98.4 & 74.9 & 122.8 & 35.7 & 15.9 & 31.2 & 79.1 \\
\hline Tianjin & -27.5 & 295.9 & -54.1 & -8.8 & 52.0 & 24.7 & -23.0 & 6.5 \\
\hline Xinjiang & 3.3 & 18.8 & 32.7 & 58.3 & 2.1 & 11.2 & 26.5 & 54.8 \\
\hline Tibet & -53.1 & 5.1 & -79.2 & -69.1 & 1.9 & 4.1 & -64.3 & -58.1 \\
\hline Yunnan & 111.4 & 93.4 & 182.8 & 84.7 & 15.6 & 13.5 & 175.6 & 75.8 \\
\hline Zhejiang & 52.6 & 144.2 & -37.6 & 74.6 & 29.9 & 26.1 & -39.4 & 66.0 \\
\hline
\end{tabular}


Table S5. Statistical performance of WRF-Chem-REAS based simulations for meteorology. Daily meteorological observations at 194 stations in 2007 are obtained from the website of National Climate Data Center of China Meteorological Administration (http://data.cma.cn/).

\begin{tabular}{ccccccccc}
\hline Meteorological items & Count & Obs. & Model & $\boldsymbol{r}$ & MB & ME & NMB & NMSE \\
\hline 2 m temperature (degree C) & 23841 & 11.51 & 9.83 & 0.97 & -1.68 & 2.43 & -14.57 & 0.1 \\
2 m relative humidity (\%) & 23862 & 61.77 & 66.1 & 0.71 & 4.34 & 12.34 & 7.02 & 0.06 \\
$10 \mathrm{~m}$ wind speed (m/s) & 23857 & 2.18 & 3.48 & 0.52 & 1.29 & 1.49 & 59.35 & 0.47 \\
\hline
\end{tabular}

Table S6. Statistical measures for seasonal variation of modeled and observed $\mathrm{PM}_{10}$ in the year 2007. Statistical indicators and associated units are described in Table 3.

\begin{tabular}{|c|c|c|c|c|c|c|c|c|c|}
\hline Month & Regions & Count & Obs & Model & $r$ & NMB & MFB & MFE & NMSE \\
\hline Jan & Central China & 176 & 147 & 142.10 & 0.30 & -3.09 & -2.02 & 35.75 & 0.18 \\
\hline Jan & Eastern China & 475 & 127 & 120.17 & 0.29 & -5.23 & -7.29 & 36.66 & 0.26 \\
\hline Jan & Northern China & 232 & 166 & 123.97 & 0.22 & -25.24 & -29.88 & 50.57 & 0.50 \\
\hline Jan & Northeastern China & 202 & 172 & 98.99 & 0.51 & -42.47 & -65.69 & 75.73 & 0.74 \\
\hline Jan & Northwestern China & 107 & 208 & 102.10 & -0.06 & -50.85 & -58.95 & 73.55 & 1.63 \\
\hline Jan & Southern China & 155 & 86 & 63.70 & 0.10 & -25.85 & -29.27 & 43.90 & 0.33 \\
\hline Jan & Southwest China & 234 & 118 & 76.82 & 0.32 & -35.02 & -52.89 & 66.07 & 0.53 \\
\hline Jan & Japan & 107 & 25 & 14.73 & 0.44 & -40.32 & -58.99 & 70.53 & 0.95 \\
\hline Jan & Nepal & 30 & 75 & 21.75 & 0.53 & -70.83 & -102.01 & 102.01 & 2.49 \\
\hline Apr & Central China & 192 & 113 & 107.56 & 0.30 & -4.95 & -5.95 & 36.89 & 0.22 \\
\hline Apr & East China & 492 & 107 & 96.38 & 0.18 & -9.78 & -10.40 & 36.60 & 0.45 \\
\hline Apr & North China & 291 & 109 & 98.65 & 0.28 & -9.14 & -13.42 & 41.69 & 0.36 \\
\hline Apr & Northeast China & 197 & 105 & 72.54 & 0.31 & -30.96 & -45.51 & 58.48 & 0.56 \\
\hline Apr & Northwest China & 129 & 135 & 139.64 & 0.26 & 3.69 & -1.88 & 47.57 & 0.58 \\
\hline Apr & South China & 106 & 84 & 65.18 & 0.23 & -22.83 & -31.27 & 48.11 & 0.35 \\
\hline Apr & Southwest China & 265 & 92 & 64.71 & 0.23 & -30.02 & -39.51 & 51.82 & 0.42 \\
\hline Apr & Japan & 104 & 37 & 21.61 & 0.77 & -42.18 & -53.85 & 61.46 & 1.46 \\
\hline Apr & Nepal & 29 & 62 & 22.47 & -0.39 & -63.56 & -82.94 & 82.94 & 1.84 \\
\hline Jul & Central China & 170 & 91 & 86.11 & 0.20 & -5.05 & -13.70 & 48.60 & 0.34 \\
\hline Jul & East China & 463 & 84 & 91.66 & 0.18 & 8.91 & 0.07 & 41.79 & 0.30 \\
\hline Jul & North China & 286 & 98 & 97.49 & 0.32 & -0.92 & -6.02 & 40.39 & 0.24 \\
\hline Jul & Northeast China & 216 & 102 & 85.54 & 0.45 & -16.14 & -30.40 & 52.68 & 0.34 \\
\hline Jul & Northwest China & 108 & 74 & 84.18 & -0.02 & 14.02 & 3.30 & 41.86 & 0.44 \\
\hline Jul & South China & 38 & 62 & 63.01 & 0.25 & 0.86 & -16.51 & 53.12 & 0.44 \\
\hline Jul & Southwest China & 195 & 74 & 47.34 & 0.05 & -35.70 & -50.11 & 60.12 & 0.58 \\
\hline Jul & Japan & 83 & 16 & 36.52 & -0.25 & 121.53 & 45.31 & 77.71 & 5.10 \\
\hline Jul & Nepal & 30 & 13 & 19.28 & 0.52 & 47.67 & 40.12 & 40.51 & 0.20 \\
\hline Oct & Central China & 188 & 119 & 120.32 & -0.02 & 1.35 & 0.18 & 41.20 & 0.29 \\
\hline Oct & East China & 478 & 94 & 101.39 & 0.33 & 7.99 & 2.51 & 37.30 & 0.22 \\
\hline Oct & North China & 259 & 101 & 104.89 & 0.22 & 4.25 & 0.98 & 43.25 & 0.32 \\
\hline Oct & Northeast China & 211 & 99 & 93.75 & 0.26 & -5.18 & -24.59 & 58.77 & 0.56 \\
\hline Oct & Northwest China & 118 & 94 & 91.94 & 0.09 & -1.68 & -12.28 & 52.02 & 0.48 \\
\hline Oct & South China & 153 & 83 & 78.42 & 0.23 & -5.93 & -10.37 & 41.00 & 0.25 \\
\hline Oct & Southwest China & 240 & 93 & 60.87 & 0.36 & -34.88 & -49.39 & 59.95 & 0.50 \\
\hline Oct & Japan & 115 & 22 & 14.48 & 0.38 & -33.57 & -44.13 & 54.72 & 0.50 \\
\hline Oct & Nepal & NA & NA & $\mathrm{NA}$ & NA & NA & NA & NA & NA \\
\hline
\end{tabular}


Table S7. Statistical measures for seasonal variation of model simulations and observation in the year 2007. The unit of Obs and Model is $\mu \mathrm{g} \mathrm{m}^{-3}$ for $\mathrm{PM}_{10}$, and $\mathrm{ppbv}$ for $\mathrm{O}_{3}, \mathrm{SO}_{2}$ and $\mathrm{NO}_{2}$. Other statistical indicators and associated units are described in Table 2.

\begin{tabular}{cccccccccc}
\hline Species & month & Count & Obs & Model & $\boldsymbol{r}$ & NMB & MFB & MFE & NMSE \\
\hline \multirow{6}{*}{$\mathrm{PM}_{10}$} & Jan & 1718 & 132.33 & 100.03 & 0.39 & -24.41 & -32.95 & 53.25 & 0.56 \\
& Apr & 1805 & 101.43 & 86.45 & 0.37 & -14.77 & -22.81 & 45.71 & 0.46 \\
& Jul & 1589 & 82.45 & 80.40 & 0.37 & -2.49 & -9.86 & 48.13 & 0.36 \\
& Oct & 1762 & 92.42 & 89.19 & 0.38 & -3.50 & -13.43 & 46.69 & 0.36 \\
\hline \multirow{6}{*}{$\mathrm{O}_{3}$} & Jan & 185 & 42.96 & 46.49 & 0.27 & 8.22 & 8.94 & 15.34 & 0.04 \\
& Apr & 181 & 60.50 & 54.31 & 0.51 & -10.23 & -9.55 & 16.87 & 0.05 \\
& Jul & 168 & 35.18 & 43.58 & 0.78 & 23.87 & 25.98 & 30.16 & 0.10 \\
& Oct & 169 & 40.59 & 47.21 & 0.43 & 16.31 & 18.20 & 25.10 & 0.08 \\
\hline \multirow{6}{*}{$\mathrm{SO}_{2}$} & Jan & 294 & 13.29 & 10.57 & 0.70 & -20.43 & -22.85 & 59.42 & 1.17 \\
& Apr & 286 & 6.67 & 7.44 & 0.70 & 11.60 & 7.69 & 61.58 & 1.25 \\
& Jul & 251 & 4.82 & 8.25 & 0.74 & 71.14 & 30.77 & 74.02 & 1.90 \\
& Oct & 269 & 5.80 & 8.91 & 0.54 & 53.67 & 24.42 & 69.58 & 2.17 \\
\hline \multirow{6}{*}{$\mathrm{NO}_{2}$} & Jan & 124 & 27.43 & 20.23 & 0.43 & -26.25 & -56.20 & 76.37 & 0.71 \\
& Apr & 120 & 22.58 & 13.43 & 0.61 & -40.52 & -67.90 & 74.07 & 0.72 \\
& Jul & 124 & 17.54 & 12.17 & 0.73 & -30.60 & -54.99 & 62.01 & 0.47 \\
& Oct & 124 & 20.56 & 14.12 & 0.53 & -31.32 & -40.78 & 66.88 & 0.68 \\
\hline
\end{tabular}

Table S8. Statistical measures for $\mathrm{O}_{3}$ at the Lulin site in different months.

\begin{tabular}{cccccccc}
\hline Month & Obs & Model & $\boldsymbol{r}$ & NMB & MFB & MFE & NMSE \\
\hline Jan & 32.97 & 48.39 & 0.63 & 46.80 & 37.99 & 37.99 & 0.17 \\
Apr & 50.80 & 53.40 & 0.38 & 5.11 & 4.95 & 17.63 & 0.05 \\
Jul & 21.14 & 35.16 & 0.48 & 66.35 & 49.60 & 49.60 & 0.33 \\
Oct & 23.40 & 44.42 & 0.35 & 89.84 & 62.80 & 62.92 & 0.51 \\
\hline
\end{tabular}




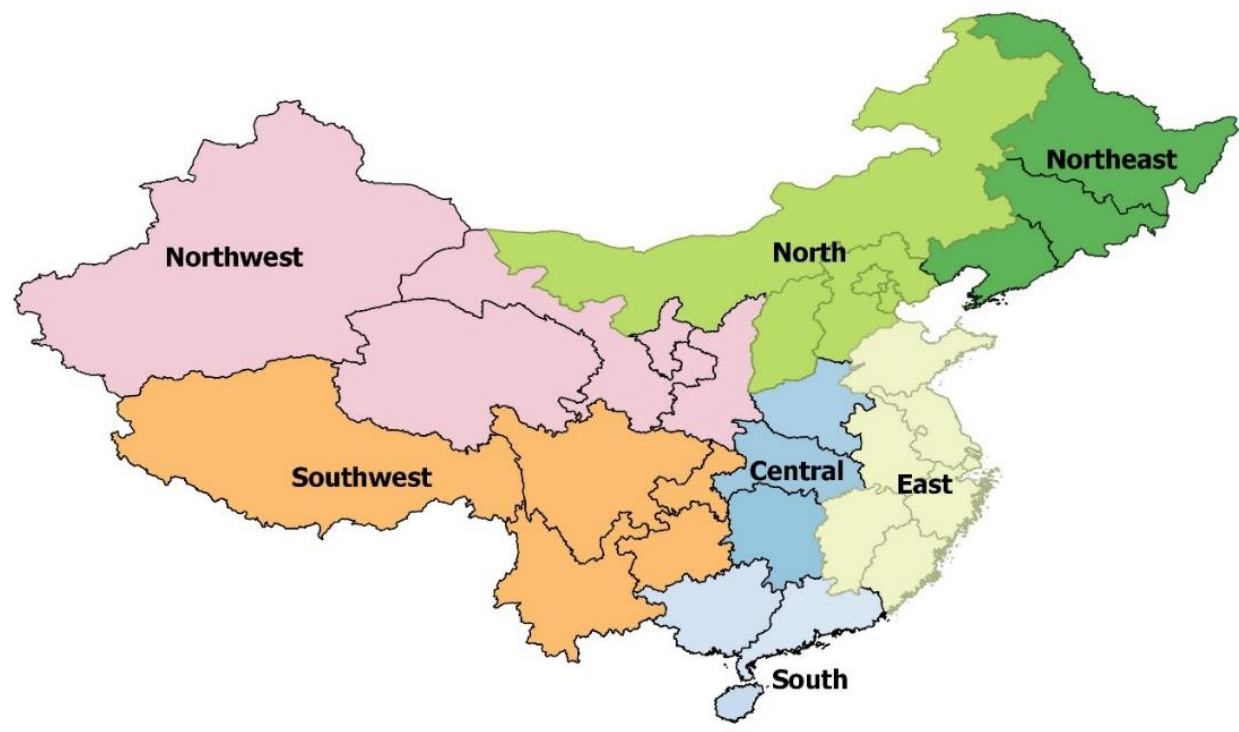

Figure S1. The seven regions in mainland China used for analysis in this paper. 


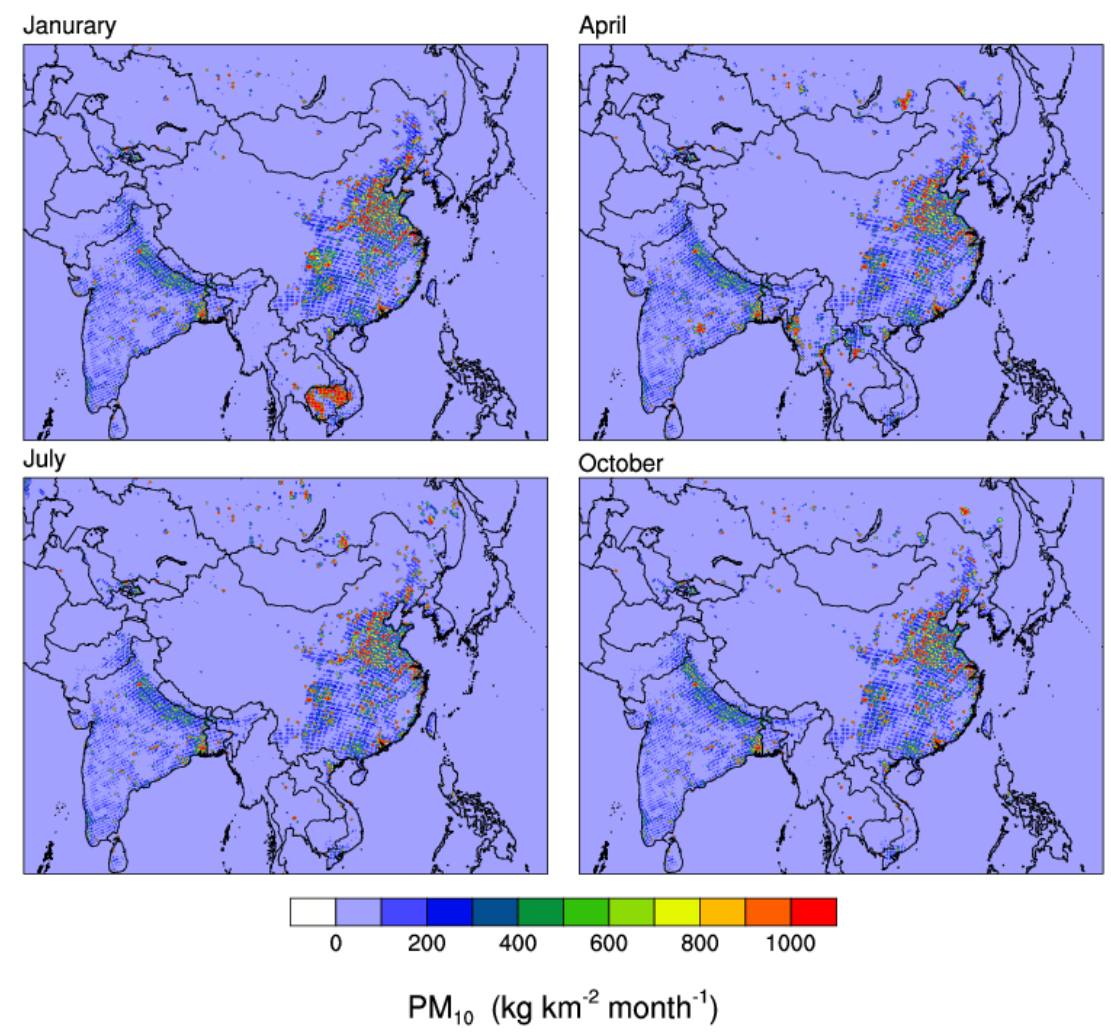

Figure S2. Monthly emissions of anthropogenic primary $\mathrm{PM}_{10}$. 

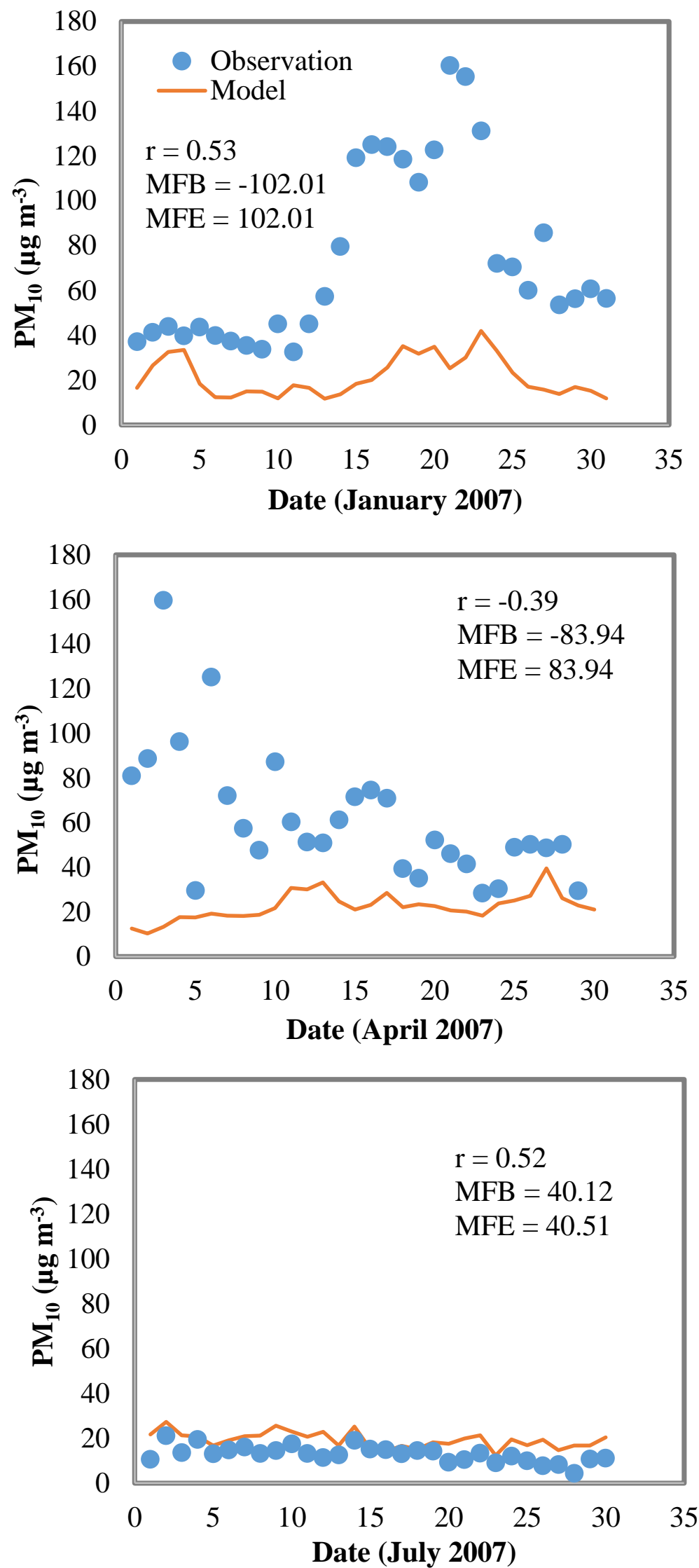

Figure S3. Comparison of observed (blue) and simulated (orange) daily mean $\mathrm{PM}_{10}$ concentrations in January (top), April (middle), and October (bottom) 2007 at Godavari , Nepal. 